\title{
Two Cases of Plug or Stone in Remnant Intrapancreatic Choledochal Cysts Treated with Endoscopic Retrograde Cholangiopancreatography
}

\author{
Eunbee Kim ${ }^{1}$, Min Ho Kang ${ }^{2}$, Jisun Lee ${ }^{2}$, Hanlim Choi ${ }^{3}$, Jae-Woon Choi ${ }^{3}$, Joung-Ho Han ${ }^{1}$ and Seon Mee Park ${ }^{1}$ \\ Department of ${ }^{1}$ Internal Medicine, ${ }^{2}$ Radiology, and ${ }^{3}$ Surgery, Chungbuk National University College of Medicine, Chungbuk, Korea
}

Incomplete resection of choledochal cysts (CCs) that extend deep into the pancreas can lead to protein plug or stone formation, pancreatitis, and cholangiocarcinoma. We encountered two cases of choledocholithiasis in remnant intrapancreatic CCs, in which the patients exhibited symptoms after 3 and 21 years of cyst excision. A 21-year-old woman who had undergone excision of a CC, as a neonate, presented with epigastric pain. Abdominal computed tomography $(\mathrm{CT})$ revealed stones inside the remnant pancreatic cyst, which were removed by endoscopic retrograde cholangiopancreatography (ERCP), and her symptoms improved. A 33-year-old woman, who underwent cyst excision 3 years ago, presented with pancreatitis. Abdominal CT showed a radiolucent plug inside the remnant pancreatic cyst. The soft, whitish plug was removed by ERCP, and the pancreatitis improved. These cases indicate that plugs and stones in CCs have the same pathogenetic mechanism, and their form depends on the time since the incomplete excision surgery. Clin Endosc 2017;50:504-507

Key Words: Choledochal cyst, intrapancreatic remnant; Cholelithiasis; Acute pancreatitis; Abdominal pain

\section{INTRODUCTION}

Choledochal cysts (CCs) are congenital cystic dilatations of the extra- or intrahepatic portion of the biliary tree, and complete excision is the gold standard treatment for these cysts. ${ }^{1}$ However, this surgery is difficult, especially when the cystic lesions extend deep into the pancreas. Injury to the pancreatic duct during operation may cause pancreatitis, stone formation, and leakage of pancreatic juice. ${ }^{2}$ Further, incomplete resection can lead to stone formation, recurrent pancreatitis, and cholangiocarcinoma in remnant intrapancreatic CCs (IPCCs). In previous studies, protein stones or plugs developed

Received: December 28, 2016 Revised: January 21, 2017

Accepted: January 24, 2017

Correspondence: Seon Mee Park

Department of Internal Medicine, Chungbuk National University College of Medicine, 1 Chungdae-ro, Seowon-gu, Cheongju 28644, Korea

Tel: +82-43-269-6019, Fax: +82-43-273-3252, E-mail: smpark@chungbuk.ac.kr

(cc) This is an Open Access article distributed under the terms of the Creative Commons Attribution Non-Commercial License (http://creativecommons.org/ licenses/by-nc/3.0) which permits unrestricted non-commercial use, distribution, and reproduction in any medium, provided the original work is properly cited. in remnant IPCCs in 5\% and 30\% patients who presented with abdominal pain or recurrent acute pancreatitis during follow-up, respectively. ${ }^{3,4}$ Complete surgical resection is recommended for such patients because of the risk of developing cholangiocarcinoma in IPCCs. ${ }^{2,5}$ However, studies have reported successful outcomes for several patients with protein stones or plugs by simply using endoscopic retrograde cholangiopancreatography (ERCP) and ensuring close follow-up. ${ }^{6,7}$

Here, we report two cases of protein stone and plug formation in remnant IPCCs, in which the patients presented with epigastric pain and acute pancreatitis. They were treated with endoscopic sphincterotomy (EST) and endoscopic papillary balloon dilation (EPBD) and followed up for 12 months, showing no recurrence.

\section{CASE REPORT}

\section{Case 1}

A 21-year-old woman was admitted to our hospital for epigastric pain. At the age of 1 month, she had undergone diver- 

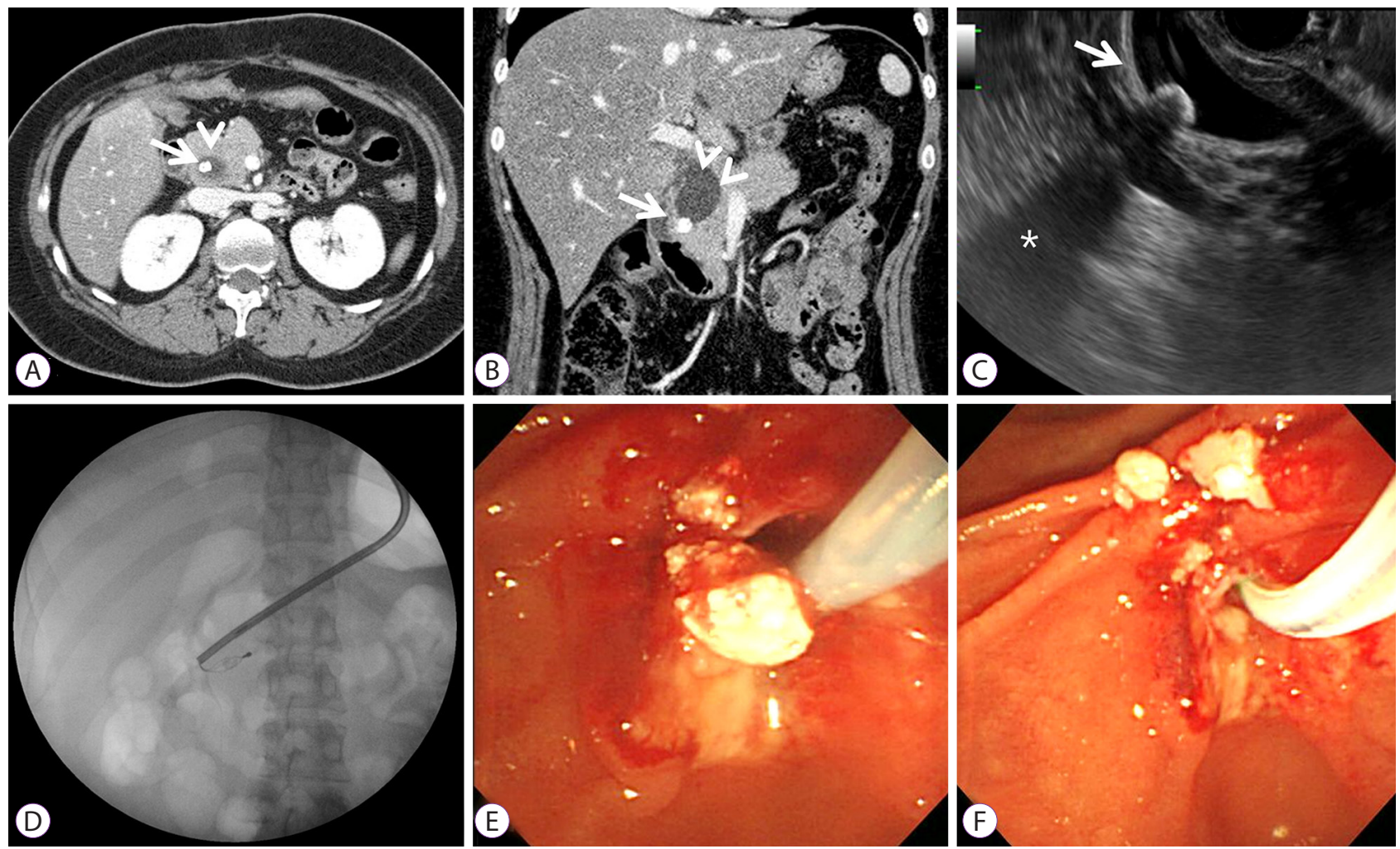

Fig. 1. Imaging of the stone in the remnant intrapancreatic choledochal cyst in case 1. (A) Axial view, (B) Coronal view. Abdominal computed tomography scan showing a round cystic lesion (arrowheads) with round stones (arrow) in the pancreatic head. (C) Endoscopic ultrasonogram showing a hypoechoic cyst (arrow) containing hyperechogenic stones with acoustic shadows (asterisks) in the pancreatic head. (D) A round stone removed using mechanical lithotripsy. (E, F) Whitish, hard protein stones removed using a basket.

sion operation for a congenital CC with biliary tract obstruction. She had been symptom free for 21 years until the current presentation. Physical examination on admission revealed tenderness at the epigastrium. Laboratory data were within normal limits, except that the patient had unconjugated hyperbilirubinemia, suggestive of Gilbert's syndrome: white blood cell count, $5,900 / \mathrm{mm}^{3}$; red blood cell count, $421 \times 10^{3} /$ $\mathrm{mm}^{3}$; hemoglobin, $12.9 \mathrm{~g} / \mathrm{dL}$; hematocrit, $37.5 \%$; platelet count, $241 \times 10^{3} / \mathrm{mm}^{3}$; total protein, $7.3 \mathrm{~g} / \mathrm{dL}$; albumin, $4.9 \mathrm{~g} / \mathrm{dL}$; aspartate aminotransferase, $31 \mathrm{U} / \mathrm{L}$; alanine aminotransferase, $49 \mathrm{U} / \mathrm{L}$; alkaline phosphatase, $64 \mathrm{U} / \mathrm{L}$; total bilirubin, $7.73 \mathrm{mg} /$ $\mathrm{dL}$; direct bilirubin, $0.44 \mathrm{mg} / \mathrm{dL}$; cholesterol, $179 \mathrm{mg} / \mathrm{dL}$; glucose, $92 \mathrm{mg} / \mathrm{dL}$; amylase, $27 \mathrm{U} / \mathrm{L}$; lipase, $46 \mathrm{U} / \mathrm{L}$. Abdominal computed tomography $(\mathrm{CT})$ revealed a $2.5 \times 4.0 \mathrm{~cm}^{2}$ circular cystic lesion with a spherical stone in the pancreatic head (Fig. 1A, B). Endoscopic ultrasonography (EUS) demonstrated a cystic lesion containing a stone inside the residual cyst (Fig. 1C). ERCP showed a round dilated distal common bile duct with filling defects, also suggesting the presence of stones in the remnant IPCC. EST and mechanical lithotripsy were performed to remove the stones (Fig. 1D), which appeared as whitish, hard, protein structures (Fig. 1E, F). The patient had an uneventful post-procedural course and was free of symp- toms, at least for the next one year.

\section{Case 2}

A 33-year-old woman was admitted to our hospital because of pain in the right upper quadrant of the abdomen. Three years previously, the patient had undergone resection of a congenital CC with anomalous union of the pancreaticobiliary duct (AUPBD). She had been symptom free until the current presentation. Physical examination at admission revealed tenderness in the epigastrium. Laboratory data were within normal limits except that the levels of amylase and lipase were elevated: white blood cell count, 9,800/ $\mathrm{mm}^{3}$; hemoglobin, $13.4 \mathrm{~g} / \mathrm{dl}$; hematocrit, $39.5 \%$; platelet count, $193 \times 10^{3} /$ $\mathrm{mm}^{3}$; total protein, $6.7 \mathrm{~g} / \mathrm{dL}$; albumin, $4.5 \mathrm{~g} / \mathrm{dL}$; aspartate aminotransferase, $17 \mathrm{U} / \mathrm{L}$; alanine aminotransferase, $14 \mathrm{U} / \mathrm{L}$; total bilirubin, $0.73 \mathrm{mg} / \mathrm{dL}$; blood glucose, $113 \mathrm{mg} / \mathrm{dl}$; amylase, 1640 U/L; lipase, 3520 IU/L. Abdominal CT and EUS showed a $3.5 \times 4.0 \mathrm{~cm}^{2}$ cystic lesion containing a radiolucent lesion in the pancreatic head (Fig. 2A-C). ERCP showed a long common channel and a cystically dilated distal common bile duct with filling defects (Fig. 2D). EST enabled easy drainage of the whitish fluid, and a protein plug, which was whitish, soft, and round, could be removed using a basket (Fig. 2E, F). After the 

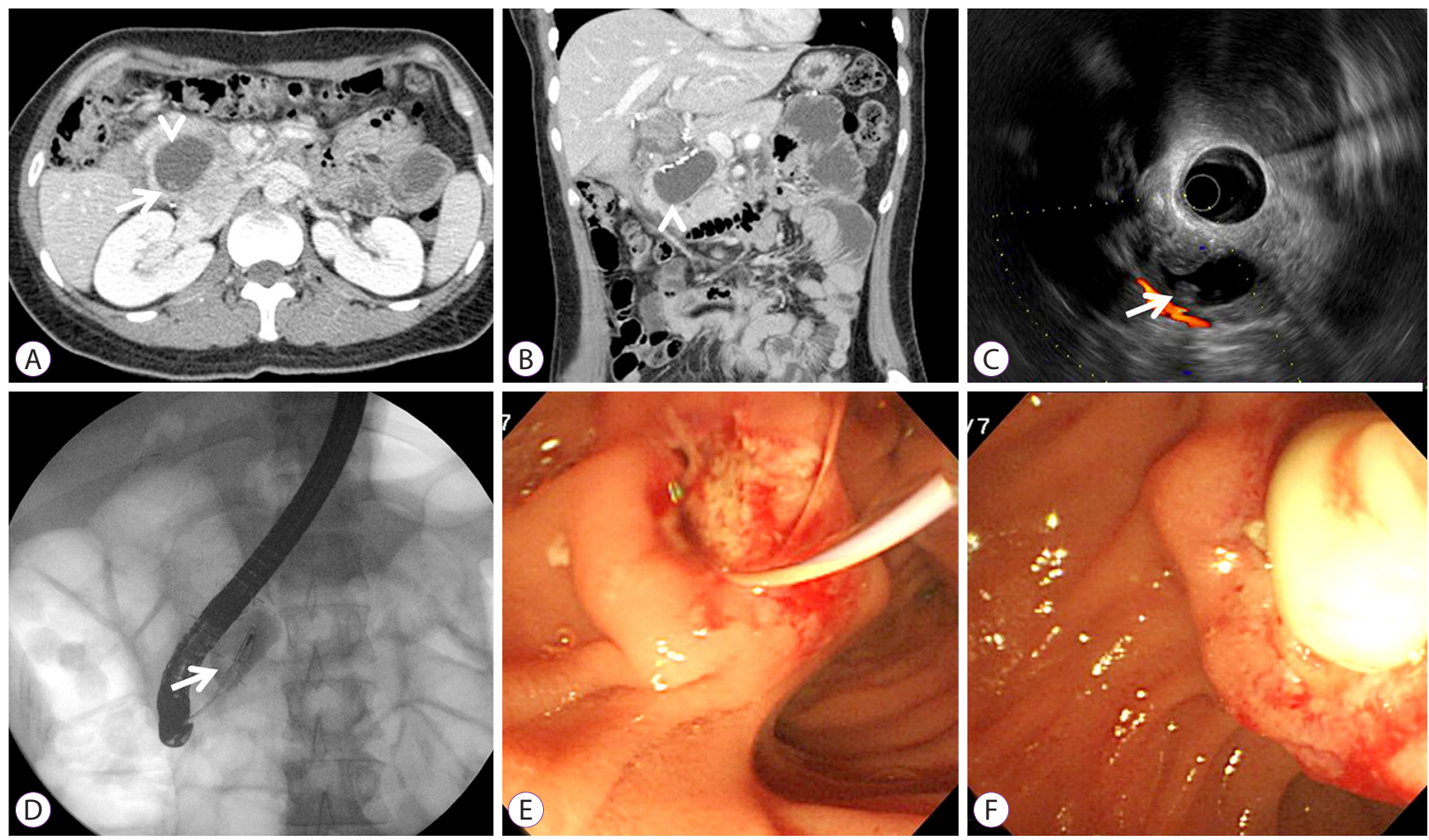

Fig. 2. Imaging of the plug in the remnant intrapancreatic choledochal cyst in case 2. (A) Axial view, (B) Coronal view. Abdominal computed tomography scan showing a round cystic lesion (arrowhead) with a round radiolucent lesion (arrow) in the pancreatic head. (C) Endoscopic ultrasonogram showing a hypoechoic cyst (arrow) containing a hyperechogenic lesion without acoustic shadows in the pancreatic head. (D) Endoscopic retrograde cholangiopancreatography showing cystic pooling with filling defects. (E, F) A soft, white plug removed using a balloon catheter and basket.

procedure, the serum amylase and lipase levels decreased, and the patient had an uneventful post-procedural course and was symptom free when discharged from the hospital. Follow-up abdominal magnetic resonance imaging showed a slight decrease in the size of the IPCC and no evidence of residual lesions in the remnant cyst.

\section{DISCUSSION}

This study showed that protein plugs and stones develop in residual IPCCs after partial excision, and they can be treated with ERCP. Whitish plugs and stones were detected in our patients at 3 and 21 years after cyst excision. We believe that plugs and stones have the same pathogenic mechanism and characteristics but that the form depends on the amount of time that has elapsed after cyst excision.

Ideally, an extrahepatic dilated bile duct and IPCCs should be completely resected during the primary operation. ${ }^{5} \mathrm{Com}$ plete excision of the extrahepatic component combined with cholecystectomy, followed by Roux-en-Y hepaticojejunosto$\mathrm{my}$, is considered the treatment of choice. Complete excision involves resection of the bile duct from the confluence of the hepatic duct proximally up to the pancreatico-biliary junction distally. ${ }^{8}$ However, when the lesion extends deep into the pancreas, complete resection is difficult, and remnant IPCCs are associated with complications such as recurrent pancreatitis, protein plug or stone formation, and malignant transformation. ${ }^{2,5}$

Protein plug or stone formation in residual IPCCs is not rare. One study reported that protein plugs and stones were detected in $28 \%-36 \%$ of IPCCs by routine intraoperative endoscopy during cyst excision. ${ }^{9}$ However, if complete cyst excision was ensured using intraoperative endoscopy, the incidence of stone formation reduced to $5.3 \%{ }^{10}$

Coexistence of an AUPBD is reported in $50 \%-80 \%$ cases of CCs. ${ }^{11}$ Reflux of protein juice to the remnant biliary cyst is the main pathogenic mechanism of protein plugs or calculi. The plausible pathogenesis of protein plug or stone formation inside IPCCs is as follows: ${ }^{12}$ The presence of a remnant IPCC leads to the formation of a dead space within the pancreas. Owing to the concomitant presence of an AUPBD, pancreatic secretion is forced into this space. If the function of the duodenal papilla is normal and duodenal juice is not regurgitated, patients who merely retain pancreatic secretions in the cyst space may not develop symptoms. However, most patients with congenital cystic dilatation of the bile duct have anatomical anomalies, and abnormal function of the duodenal papilla 
results in backflow of intestinal secretions, which activates pancreatic enzymes in the remnant cyst, leading to infection, formation of intrabiliary carcinogens, and an increased risk of malignancy.

We suggest that incomplete resection of CC during the primary operation and the increase in intraluminal pressure in the pancreatic duct caused by the dynamic obstruction produced by a protein plug or pancreatic stone play an important role in dilatation of the choledochal remnant after congenital CC excision.

Chemical and infrared spectrometric analyses of stones showed that albumin (more than 98\%) and calcium (0.53\%) were the main components, indicating that the composition was different from that of typical pancreatic and bile duct stones. ${ }^{7}$ Protein plugs are white and fragile and comprise over $90 \%$ protein with concentrated pancreatic juice. ${ }^{3,4}$ In the present study as well, the plugs were whitish and fragile, appeared radiolucent in abdominal CT scans, and showed no acoustic shadowing on EUS. In contrast, the stones were white and hard and produced an acoustic shadowing.

The treatment options for residual IPCCs are reoperation for complete resection, close observation, and endoscopic treatment. Most studies showed that radical excision of the dilated cystic remnant, buried in the pancreatic head, was associated with low morbidity rates and yielded favorable longterm outcomes. ${ }^{12}$ The efficacy of endoscopic removal of protein plugs/stones remains debatable. ERCP has been reported as a useful technique to treat acute pancreatitis induced by remnant cysts. ${ }^{4}$ However, a study reported recurrent stones in and pancreatic juice reflux to IPCCs after EST, indicating that complete resection of the cysts is mandatory to prevent future problems. ${ }^{6}$ We believe that in our patients, EST and/or EPBD widened the common channel, so close follow-up for more than 1 year was mainly needed.

The risk of malignant transformation after incomplete excision of IPCCs should be carefully considered. The incidence of metachronous carcinoma was found to depend on resection completeness, and it is reportedly $0.7 \%{ }^{11}-11.3 \%{ }^{12}$ in the case of incomplete resection and $20 \%-30 \%$ in the case of untreated CCs. ${ }^{11}$ Additionally, a study showed that this risk at 15,20 , and 25 years after cyst excision was $1.6 \%, 3.9 \%$, and $11.3 \%$, respectively. ${ }^{12}$ One study reported that the average time between incomplete CC excision and the diagnosis of malignancy was 140 months. ${ }^{13}$ The risk of malignancy is also related to the type of CCs: it is higher for types I and IV than it is for types II, III, and V. ${ }^{1}$

Our patients, both young women, refused re-operation for complete resection and preferred close follow-up. There is currently no consensus or recommendation on the proper follow-up interval and methods. Long-term follow-up for bile duct cancer is widely accepted, using abdominal CT scan, pancreatic endosonography, or magnetic resonance cholangiopancreatography and laboratory investigations including liver function parameters and tumor markers (carcinoembryonic antigen [CEA], carbohydrate antigen [CA] 19-9, and cancer antigen [CA]-125) conducted every 6-12 months. ${ }^{11}$

In conclusion, through this report on two cases of protein plugs and stones, we attempt to explain the process of stone formation in remnant IPCCs. Reoperation for complete resection is recommended in such cases. If not, symptomatic treatment with ERCP and close surveillance for cholangiocarcinoma are mandatory.

\section{Conflicts of Interest}

The authors have no financial conflicts of interest.

\section{REFERENCES}

1. Todani T, Tabuchi K, Watanabe Y, Kobayashi T. Carcinoma arising in the wall of congenital bile duct cysts. Cancer 1979;44:1134-1141.

2. Cheung TT, Fan ST. Technical note on complete excision of choledochal cysts. Hepatobiliary Pancreat Dis Int 2013;12:218-221.

3. Kaneko K, Ando H, Ito T, et al. Protein plugs cause symptoms in patients with choledochal cysts. Am J Gastroenterol 1997;92:1018-1021.

4. Chiba K, Kamisawa T, Egawa N. Relapsing acute pancreatitis caused by protein plugs in a remnant choledochal cyst. J Hepatobiliary Pancreat Sci 2010;17:729-730.

5. Jordan PH Jr, Goss JA Jr, Rosenberg WR, Woods KL. Some considerations for management of choledochal cysts. Am J Surg 2004;187:790795.

6. Hsu RK, Yu A, Lee JG, Leung JW. Pancreatitis caused by common bile duct stones in a 3-year-old boy with prior surgery for a choledochal cyst. Am J Gastroenterol 2001;96:1919-1921.

7. Nakano K, Mizuta A, Oohashi S, et al. Protein stone formation in an intrapancreatic remnant cyst after resection of a choledochal cyst. Pancreas 2003;26:405-407.

8. Kim JW, Moon SH, Park DH, et al. Course of choledochal cysts according to the type of treatment. Scand J Gastroenterol 2010;45:739-745.

9. Takahashi T, Shimotakahara A, Okazaki T, et al. Intraoperative endoscopy during choledochal cyst excision: extended long-term follow-up compared with recent cases. J Pediatr Surg 2010;45:379-382.

10. Urushihara N, Fukumoto K, Fukuzawa H, et al. Long-term outcomes after excision of choledochal cysts in a single institution: operative procedures and late complications. J Pediatr Surg 2012;47:2169-2174.

11. Jabłońska B. Biliary cysts: etiology, diagnosis and management. World J Gastroenterol 2012;18:4801-4810.

12. Ohashi T, Wakai T, Kubota M, et al. Risk of subsequent biliary malignancy in patients undergoing cyst excision for congenital choledochal cysts. J Gastroenterol Hepatol 2013;28:243-247.

13. Xia HT, Yang T, Liang B, Zeng JP, Dong JH. Treatment and outcomes of adults with remnant intrapancreatic choledochal cysts. Surgery 2016;159:418-425. 